\title{
Geometry of Mutation Classes of Rank 3 Quivers
}

\author{
Anna Felikson ${ }^{1} \cdot$ Pavel Tumarkin ${ }^{1}$
}

Received: 18 September 2018 / Revised: 16 February 2019 / Accepted: 21 February 2019 /

Published online: 4 March 2019

(c) The Author(s) 2019

\begin{abstract}
We present a geometric realization for all mutation classes of quivers of rank 3 with real weights. This realization is via linear reflection groups for acyclic mutation classes and via groups generated by $\pi$-rotations for the cyclic ones. The geometric behavior of the model turns out to be controlled by the Markov constant $p^{2}+q^{2}+r^{2}-p q r$, where $p, q, r$ are the weights of arrows in a quiver. We also classify skew-symmetric mutation-finite real $3 \times 3$ matrices and explore the structure of acyclic representatives in finite and infinite mutation classes.
\end{abstract}

Keywords Quiver mutation · Reflection · Markov constant

Mathematics Subject Classification $13 \mathrm{~F} 60 \cdot 20 \mathrm{H} 15 \cdot 51 \mathrm{~F} 15$

\section{Introduction and Main Results}

Mutations of quivers were introduced by Fomin and Zelevinsky (2002) in the context of cluster algebras and since then have found numerous applications in various domains of mathematics. Mutations are involutive transformations decomposing the set of quivers into equivalence classes called mutation classes (see Sect. 2.1 for precise definitions). Knowing the structure of mutation classes gives a lot of information about the corresponding cluster algebras. It is especially beneficial if there exists a certain combinatorial or geometric model for mutations. This is the case, for example,

To Rafail Kalmanovich Gordin on his 70th birthday.

Anna Felikson was partially supported by EPSRC Grant EP/N005457/1.

Pavel Tumarkin

pavel.tumarkin@durham.ac.uk

Anna Felikson

anna.felikson@durham.ac.uk

1 Department of Mathematical Sciences, Durham University, Science Laboratories, South Road, Durham DH1 3LE, UK 
for adjacency quivers of triangulations of bordered marked surfaces (Fock and Goncharov 2006; Gekhtman et al. 2005; Fomin et al. 2008), where mutations correspond to flips of triangulations.

There is a model for mutations of quivers containing a representative without oriented cycles in their mutation class (such quivers are called mutation-acyclic): it was shown in (Speyer and Thomas 2013; Seven 2015) that mutations of mutation-acyclic quivers can be modeled by reflections of a tuple of positive vectors in a certain quadratic space (we call this a realization by reflections). One of the goals of this paper is to construct a model for mutations of mutation-cyclic quivers of rank 3 , and thus to obtain a geometric realization for every quiver of rank 3 (Theorem 3.6). In particular, we prove that mutations of mutation-cyclic rank 3 quivers can be modeled by $\pi$-rotations in triples of points on a hyperbolic plane.

Mutation classes of rank 3 quivers were studied in Assem et al. (2008), Beineke et al. (2011), Berenstein et al. (2006), Felikson et al. (2012), Seven (2012) and Warkentin (2014). In particular, the Markov constant $C(Q)=p^{2}+q^{2}+r^{2}-p q r$ for a cyclic quiver $Q$ with weights ( $p, q, r)$ was introduced in Beineke et al. (2011) and proved to be mutation-invariant. Combining our results with ones of Beineke et al. (2011), we show that $C(Q)$ defines the type and geometric properties of realizations of all rank 3 quivers (Theorem 4.4). For mutation-acyclic quivers, $C(Q)$ also controls the signature of the quadratic space where mutations are modeled by reflections. More precisely, after considering appropriate projectivization, $C(Q)$ chooses between the sphere $\mathbb{S}^{2}$, Euclidean plane $\mathbb{E}^{2}$ and the hyperbolic plane $\mathbb{H}^{2}$, see Remark 4.7.

Throughout the whole paper, we allow a quiver to have real weights, so all the results concern a more general class of quivers than is usually considered (see also Lampe 2018). A quiver is mutation-finite if its mutation class is finite. The classification of mutation-finite quivers with integer weights in rank 3 is extremely simple: there are two quivers in the mutation class of an orientation of $A_{3}$ Dynkin diagram, two quivers in the mutation class of an acyclic orientation of $A_{2}^{(1)}$ extended Dynkin diagram, and the Markov quiver. However, in the case of real weights the question is more interesting, we classify all the finite mutation classes in rank 3 in Theorem 5.9, which, in its turn, leads to the complete classification of mutation-finite quivers with real weights (Felikson and Tumarkin 2019).

Finally, we discuss the structure of acyclic representatives in mutation classes. According to Caldero and Keller (2006), all acyclic quivers in any integer mutation class can be mutated to each other via sink-source mutations only, i.e. by mutations in vertices incident to incoming (or outgoing) arrows only. This is not the case for quivers with real weights: already finite mutation classes may have two essentially distinct acyclic representatives (see Table 1), and infinite mutation classes have infinitely many ones which are distributed densely, see Theorem 6.2.

\section{Mutation-Acyclic Quivers via Reflections}

In this section we model mutations of a mutation-acyclic rank 3 quiver via some linear reflection group acting on $\mathbb{S}^{2}, \mathbb{E}^{2}$ or $\mathbb{H}^{2}$. The results of this section can be 
deduced from Barot et al. (2006) [see also Seven (2015), Speyer and Thomas (2013) and Felikson and Tumarkin (2018) for more general picture], we give a geometric interpretation and observe that taking real weights instead of integer ones does not affect the proofs.

\subsection{Quiver Mutations}

A quiver $Q$ is an oriented graph with weighted edges without loops, 2-cycles and multiple edges. We allow the weights to be any positive real numbers. We call the directed edges arrows. By rank of $Q$ we mean the number of its vertices.

For every vertex $k$ of $Q$ we define an involutive operation $\mu_{k}$ called mutation of $Q$ in direction $k$. It gives a new quiver $\mu_{k}(Q)$ which can be obtained from $Q$ in the following way (see Fomin and Zelevinsky 2002):

- orientations of all arrows incident to the vertex $k$ are reversed, weights remain intact;

- for every pair of vertices $(i, j)$ such that $Q$ contains arrows directed from $i$ to $k$ and from $k$ to $j$ the weight of the arrow joining $i$ and $j$ changes as described in Fig. 1.

Given a quiver $Q$, its mutation class is a set of all quivers (considered up to isomorphism) obtained from $Q$ by all sequences of iterated mutations. All quivers from one mutation class are called mutation-equivalent.

Quivers without loops and 2-cycles are in one-to-one correspondence with real skew-symmetric matrices $B=\left\{b_{i j}\right\}$, where $b_{i j}>0$ if and only if there is an arrow from $i$ th vertex to $j$ th one with weight $b_{i j}$. In terms of the matrix $B$ the mutation $\mu_{k}$ can be written as $\mu_{k}(B)=B^{\prime}$, where

$$
b_{i j}^{\prime}= \begin{cases}-b_{i j}, & \text { if } i=k \text { or } j=k \\ b_{i j}+\frac{\left|b_{i k}\right| b_{k j}+b_{i k}\left|b_{k j}\right|}{2}, & \text { otherwise. }\end{cases}
$$

A rank 3 quiver (and the corresponding $3 \times 3$ matrix) is called cyclic if its arrows compose an oriented cycle, and is called acyclic otherwise. A quiver (and the matrix) is mutation-cyclic if all representatives of the mutation class are cyclic, and mutationacyclic otherwise.

Fig. 1 Quiver mutations. The sign before $r$ (resp., $r^{\prime}$ ) is positive if the vertices of $Q$ (resp., $Q^{\prime}$ ) form an oriented cycle, and negative otherwise. Either $r$ or $r^{\prime}$ may vanish

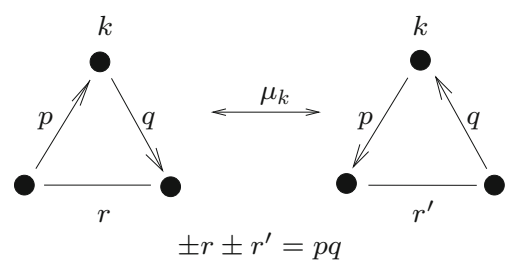




\subsection{Construction}

\subsubsection{Initial Configuration}

Let $Q$ be an acyclic rank 3 quiver and let $B$ be the corresponding skew-symmetric $3 \times 3$ matrix. Consider a symmetric matrix with non-positive off-diagonal entries $M(B)=\left(m_{i j}\right)$, where $m_{i i}=2, m_{i j}=-\left|b_{i j}\right|$ if $i \neq j$.

$M(B)$ defines a quadratic form, and we can consider it as the matrix of inner products of some triple of vectors $\left(v_{1}, v_{2}, v_{3}\right)$ in a quadratic space $V$ of the same signature as $M(B)$ has. Considering the projectivization $P(V)=V / \mathbb{R}_{+}$, the images $l_{i}$ of the hyperplanes $\Pi_{i}=v_{i}^{\perp}$ define lines in a space $X$ of constant curvature, where $X$ is the sphere $\mathbb{S}^{2}$ if $M(B)$ is positive definite, a Euclidean plane $\mathbb{E}^{2}$ if $M(B)$ is degenerate positive semidefinite, or $\mathbb{H}^{2}$ if $M(B)$ is of signature $(2,1)$. The scalar product $\left(v_{i}, v_{j}\right)$ characterizes the mutual position of the corresponding lines:

$$
\left|\left(v_{i}, v_{j}\right)\right|= \begin{cases}2 \cos \angle\left(\Pi_{i}, \Pi_{j}\right)<2 & \text { if } l_{i} \text { intersects } l_{j}, \\ 2 & \text { if } l_{i} \text { is parallel to } l_{j} \\ 2 \cosh d\left(l_{i}, l_{j}\right)>2 & \text { otherwise }\end{cases}
$$

where $d\left(l_{i}, l_{j}\right)$ is the distance between diverging lines in $\mathbb{H}^{2}$.

Consider also the halfplanes $l_{i}^{-}=\left\{u \in P(V) \mid\left(u, v_{i}\right)<0\right\}$, let $F=l_{1}^{-} \cap l_{2}^{-} \cap l_{3}^{-}$. Since $\left(v_{i}, v_{j}\right) \leq 0, F$ is an acute-angled domain (i.e., $F$ has no obtuse angles).

\subsubsection{Reflection Group}

Given a vector $v_{i} \in V$ with $\left(v_{i}, v_{i}\right)=2$ one can consider a reflection with respect to $l_{i}=v_{i}^{\perp}$ defined by $r_{i}(u)=u-\left(u, v_{i}\right) v_{i}$. Reflections preserve the scalar product in $V$, and $r_{i}\left(v_{i}\right)=-v_{i}$, i.e. $r_{i}$ is an isometry of $X$ preserving $l_{i}$ and interchanging the halfspaces into which $X$ is decomposed by $l_{i}$. We denote by $G$ the group generated by reflections $r_{1}, r_{2}, r_{3}$.

\subsubsection{Mutation}

The initial acyclic quiver $Q$ (and matrix $B$ ) corresponds to the initial set of generating reflections in the group $G$ and to the initial domain $F \subset P(V)$. Applying mutations, we will obtain other sets of generating reflections in $G$ as well as other domains in $P(V)$.

More precisely, define mutation of the set of generating reflections by partial conjugation: $\mu_{k}\left(r_{j}\right)=r_{k} r_{j} r_{k}$ if $b_{j k}>0$, and $\mu_{k}\left(r_{j}\right)=r_{j}$ otherwise. Consequently, the mutation of the triple of vectors (and of the triple of lines) is defined by partial reflection:

$$
\mu_{k}\left(v_{j}\right)= \begin{cases}v_{j}-\left(v_{j}, v_{k}\right) v_{k} & \text { if } b_{j k}>0 \\ -v_{k} & \text { if } j=k \\ v_{j} & \text { otherwise }\end{cases}
$$


Note that the mutation as defined above is not an involution. To fix this, choose a vector $u \in F$ and define $\mu_{k}$ as above (i.e. reflecting $v_{j}$ if $b_{j k}>0$ ) for the case $\left(u, v_{k}\right)<0$, and by reflection of $v_{j}$ if $b_{j k}<0$ for the case $\left(u, v_{k}\right)>0$. Applications of two versions of the definition differ by reflection in $v_{k}$ only. Throughout the paper we will mostly use the configurations up to conjugation by an element of $G$, so it will be sufficient for us to use the initial definition.

\subsection{Geometric Realization by Reflections}

Lemma 2.1 (Barot et al. (2006), Corollary of Proposition 3.2) Let $Q$ be a rank 3 quiver, and let $B$ be the corresponding skew-symmetric matrix. Let $V=\left\langle v_{1}, v_{2}, v_{3}\right\rangle$ be a quadratic space and suppose that

(1) $\left(v_{i}, v_{i}\right)=2$ for $i=1,2,3,\left|\left(v_{i}, v_{j}\right)\right|=\left|b_{i j}\right|$ for $1 \leq i<j \leq 3$;

(2) if $\left(v_{i}, v_{j}\right) \neq 0$ for all $i \neq j$, then the number of pairs $(i, j)$ such that $i<j$ and $\left(v_{i}, v_{j}\right)>0$ is even if $Q$ is acyclic and odd if $Q$ is cyclic.

Then the set of vectors $\mathbf{v}^{\prime}=\left(\mu_{k}\left(v_{1}\right), \mu_{k}\left(v_{2}\right), \mu_{k}\left(v_{3}\right)\right)$ satisfies conditions (1)-(2) for $B^{\prime}=\mu_{k}(B)$.

We note that the statement of (Barot et al. 2006, Proposition 3.2) is formulated in terms of quasi-Cartan companions, which are Gram matrices of tuples of vectors $\left\{v_{1}, v_{2}, v_{3}\right\}$, and their mutations, which are precisely changes of bases corresponding to our mutations $\mathbf{v} \mapsto \mathbf{v}^{\prime}$ defined above.

The statement of the lemma is proved in Barot et al. (2006) for integer skewsymmetrizable matrices, however, their proof works for real skew-symmetric matrices as well. One can also note that for any skew-symmetric matrix $B$ there exists a quadratic three-dimensional space $V$ and a triple of vectors $v_{1}, v_{2}, v_{3} \in V$ satisfying the assumptions of the lemma.

Definition 2.2 Let $B$ be a $3 \times 3$ skew-symmetric matrix. We say that a tuple of vectors $\mathbf{v}=\left(v_{1}, v_{2}, v_{3}\right)$ is a geometric realization by reflections of $B$ if conditions (1)-(2) of Lemma 2.1 are satisfied. We also say that $\mathbf{v}$ provides a realization of the mutation class of $B$ if the mutations of $\mathbf{v}$ via partial reflections agree with the mutations of $B$, i.e. if conditions (1)-(2) are satisfied after every sequence of mutations.

Given a geometric realization $\left(v_{1}, v_{2}, v_{3}\right)$ of $B$, consider the lines $l_{i}=\left\{u \mid\left(u, v_{i}\right)=\right.$ $0\}$. The (unordered) triple of lines $\left(l_{1}, l_{2}, l_{3}\right)$ will be also called a geometric realization by reflections of $B$ (note that properties (1)-(2) do not depend on the choice of vectors orthogonal to $\left.\left(l_{1}, l_{2}, l_{3}\right)\right)$. A realization of $B$ will also be called a realization of the corresponding quiver $Q$.

Corollary 2.3 Every acyclic mutation class has a geometric realization by reflections.

Proof In view of Lemma 2.1 it is sufficient to find a geometric realization for an acyclic quiver. This is provided by the construction above (notice that for the initial acyclic quiver we get $\left(v_{i}, v_{j}\right)<0$, so condition (2) holds). 
Remark 2.4 In contrast to quivers with integer weights, mutation classes of quivers with real weights may have more than one acyclic representative (modulo sink-source mutations), we discuss this in the last section. Meanwhile, we observe that by Lemma 2.1 a triple of lines corresponding to any acyclic quiver determines an acute-angled domain, while a triple corresponding to a cyclic quiver determines a domain with an obtuse angle.

\section{Mutation-Cyclic Quivers via $\pi$-Rotations}

\subsection{Construction}

Similarly to acyclic mutation classes realized by partial reflections in $\mathbb{S}^{2}, \mathbb{E}^{2}$ or $\mathbb{H}^{2}$, we will use $\pi$-rotations in $\mathbb{H}^{2}$ to build a geometric realization for mutation-cyclic classes.

\subsubsection{Initial Configuration}

Let $Q$ be a cyclic rank 3 quiver and let $B$ be the corresponding skew-symmetric $3 \times 3$ matrix (we will assume $b_{12}, b_{23}, b_{31}>0$ ). We will also assume $\left|b_{i j}\right| \geq 2$ for all $i \neq j$ (in view of Lemma 3.3 below this is the case for quivers in mutation-cyclic classes).

Let $V$ be a quadratic space of signature $(2,1)$, suppose that $v_{1}, v_{2}, v_{3}$ are negative vectors with $\left(v_{i}, v_{i}\right)=-2,\left|\left(v_{i}, v_{j}\right)\right|=\left|b_{i j}\right|$ for $i \neq j$. Then $v_{i}$ correspond to points in the hyperbolic plane $\mathbb{H}^{2}$, the product $\left(v_{i}, v_{j}\right)$ represents the distance $d\left(v_{i}, v_{j}\right)$, i.e., $\left(v_{i}, v_{j}\right)=-2 \cosh d\left(v_{i}, v_{j}\right)$.

It is not immediately evident that for every mutation-cyclic matrix $B$ there is a corresponding triple of vectors $v_{1}, v_{2}, v_{3}$, we will prove this in Sect. 4.

\subsection{2 $\pi$-Rotations Group}

With every $x \in \mathbb{H}^{2}$ (i.e., with every negative $v \in V$ ) we can associate a rotation by $\pi$ around $x$. A $\pi$-rotation $R_{v}$ about $v,(v, v)=-2$, acts as $R_{v}(u)=-u-(u, v) v$. Given three points $v_{1}, v_{2}, v_{3}$, we can generate a group $G=\left\langle R_{v_{1}}, R_{v_{2}}, R_{v_{3}}\right\rangle$ acting on $\mathbb{H}^{2}$.

\subsubsection{Mutation}

The initial matrix $B$ corresponds to the initial set of generating rotations in the group $G$ and to the initial triple of points in $\mathbb{H}^{2}$. Applying mutations, we will obtain other sets of generating rotations of $G$ as well as other triples of points.

More precisely, define mutation of the set of generating rotations by partial conjugation, in exactly the same way as for reflections: $\mu_{k}\left(r_{j}\right)=r_{k} r_{j} r_{k}$ if $b_{j k}>0$, and $\mu_{k}\left(r_{j}\right)=r_{j}$ otherwise. Consequently, the mutation of the triple of points is defined by partial rotation:

$$
\mu_{k}\left(v_{j}\right)= \begin{cases}-v_{j}-\left(v_{j}, v_{k}\right) v_{k} & \text { if } b_{j k}>0 \\ v_{i} & \text { otherwise }\end{cases}
$$




\subsection{Geometric Realization by $\pi$-Rotations}

Lemma 3.1 Let $Q$ be a cyclic quiver of rank 3 with all weights greater or equal to 2 , let $B$ be the corresponding skew-symmetric matrix with $b_{12}, b_{23}, b_{31}>0$, and let $V$ be the corresponding quadratic space. Suppose that $v_{1}, v_{2}, v_{3} \in V$ are vectors satisfying $\left(v_{i}, v_{i}\right)=-2,\left(v_{i}, v_{j}\right)=-\left|b_{i j}\right|$ for $1 \leq i<j \leq 3$.

Then $Q^{\prime}=\mu_{k}(Q)$ is a cyclic quiver with weights greater or equal to 2 , and the set of vectors $\mathbf{v}^{\prime}=\left(\mu_{k}\left(v_{1}\right), \mu_{k}\left(v_{2}\right), \mu_{k}\left(v_{3}\right)\right)$ satisfies the assumptions of the lemma for $B^{\prime}=\mu_{k}(B)$.

Proof Due to the symmetry, to prove the lemma we only need to check one mutation (say, $\left.\mu_{2}\right)$. A direct computation shows that $\left(v_{1}^{\prime}, v_{3}^{\prime}\right)=-\left(b_{12} b_{23}-b_{31}\right)=-b_{13}^{\prime}$, $\left(v_{1}^{\prime}, v_{2}^{\prime}\right)=\left(v_{1}, v_{2}\right)=b_{12}^{\prime},\left(v_{2}^{\prime}, v_{3}^{\prime}\right)=\left(v_{2}, v_{3}\right)=b_{23}^{\prime}$. As $v_{1}^{\prime}$ and $v_{3}^{\prime}$ are negative, $\left(v_{1}^{\prime}, v_{3}^{\prime}\right)=-2 \cosh d\left(v_{1}^{\prime}, v_{3}^{\prime}\right)<-2<0$, which implies that $b_{31}^{\prime}=-b_{13}^{\prime}<-2$, i.e. $Q^{\prime}=\mu_{2}(Q)$ is a cyclic quiver with $\left|b_{12}^{\prime}\right|,\left|b_{23}^{\prime}\right|,\left|b_{31}^{\prime}\right| \geq 2$ for $B^{\prime}=\mu_{2}(B)$. Also, the computation above shows that the assumptions are satisfied by $\mathbf{v}^{\prime}$ and $B^{\prime}$.

Notation 3.2 From now on, given a cyclic quiver we denote its weights by $p=\left|b_{12}\right|$, $q=\left|b_{23}\right|, r=\left|b_{31}\right|$. We will also denote the corresponding matrix $B$ by a triple $(p, q, r)$.

A quiver is called minimal if the sum of its weights is minimal across the whole mutation class.

Lemma 3.3 Let $Q$ be a cyclic quiver with weights $p, q, r>0$.

(a) if $r<2$ then $Q$ is mutation-acyclic;

(b) if $r=2$ and $p \neq q$ then $Q$ is mutation-acyclic;

(c) if $r=2$ and $p=q \geq 2$ then $Q$ is mutation-cyclic, and $Q$ is minimal in its mutation class.

Proof (a) We will apply mutations $\mu_{1}$ and $\mu_{3}$ alternately (starting from $\mu_{3}$ ), so that at every step $b_{13}=r$ stays intact. Furthermore, each of the steps changes either $b_{12}$ or $b_{23}$ as follows:

Claim 1 For $n \in \mathbb{N}$ denote $Q_{n}^{\prime}=\left(\mu_{1} \mu_{3}\right)^{n / 2} Q$ ifn is even or $Q_{n}^{\prime}=\mu_{3}\left(\mu_{1} \mu_{3}\right)^{(n-1) / 2} Q$ if $n$ is odd. If all $Q_{k}^{\prime}$ are cyclic for $k<n$, then the entries of the corresponding matrix $B_{n}^{\prime}$ satisfy

$$
\left|b_{12}^{\prime}\right|\left(\text { or }\left|b_{23}^{\prime}\right|\right)=f_{n}(p, q, r)=u_{n}(r) q-u_{n-1}(r) p,
$$

where $u_{n}(x)$ is a Chebyshev polynomial of the second kind (of a half-argument) recursively defined by $u_{0}(x)=1, u_{1}(x)=x, u_{n+1}(x)=x u_{n}(x)-u_{n-1}(x)$.

The proof is an easy induction: $\mu_{3}(p, q, r)=(r q-p, q, r)$, and the step is given by $\mu=\mu_{1}$ or $\mu_{3}$ with $\mu\left(f_{n}, f_{n+1}, r\right)=\left(f_{n+2}, f_{n+1}, r\right)$. The claim can also be extracted from (Lee and Schiffler (2015), Lemma 3.2).

Claim 2 For any real $p, q, r>0$ s.t. $r<2$ there exists $n \in \mathbb{Z}_{+}$such that $u_{n+1}(r) q-$ $u_{n}(r) p<0$. 
To prove the claim, we will use Chebyshev polynomials of the second kind defined by

$$
U_{0}(y)=1, \quad U_{1}(y)=2 y, \quad U_{n+1}(y)=2 y U_{n}(y)-U_{n-1}(y) .
$$

Notice that if $x=2 y$ then $u_{n}(x)=U_{n}(y)$. For $0<r<2$ we can write $r=2 \cos \theta$ for some $0<\theta<\pi / 2$. Then we have

$$
u_{n}(r)=U_{n}(\cos \theta)=\frac{\sin ((n+1) \theta)}{\sin \theta},
$$

where the last equality is a well-known property of Chebyshev polynomials of the second kind. If $u_{n+1}(r) q-u_{n}(r) p \geq 0$, then

$$
\frac{\sin ((n+1) \theta)}{\sin \theta} q \geq \frac{\sin (n \theta)}{\sin \theta} p,
$$

or just $\sin ((n+1) \theta) q \geq \sin (n \theta) p$, as $\sin \theta>0$. Since $0<\theta<\pi / 2$, there exists $n>0$ such that $\sin (k \theta)>0$ for all $0<k \leq n$ but $\sin ((n+1) \theta)<0$. This gives the number $n$ required in Claim 2.

Combining the two claims we see that there exists $n \in \mathbb{N}$ such that $Q_{n}^{\prime}$ is acyclic, which completes the proof of part (a).

(b) If $r=2$ then $u_{n}(r)=n+1$, so, the condition $u_{n+1}(r) q-u_{n}(r) p>0$ turns into $(n+1) q-n p>0$. Assuming $q<p$, this cannot hold if $n$ is large enough.

(c) If $p=q>2$ and $r=2$ then there exist points $v_{1}, v_{2}, v_{3}$ in $\mathbb{H}^{2}$ realizing $B=$ $(q, q, r)$. Indeed, we take $v_{1}=v_{3}$, and choose any $v_{2}$ such that $2 \cosh d\left(v_{1}, v_{2}\right)=q$ (as usual, we assume $\left(v_{i}, v_{i}\right)=-2$ ). Applying repeatedly Lemma 3.1 we see that in this case $Q$ is mutation-cyclic. Moreover, the mutated triple of points always remains collinear, and it is easy to see that every new mutation either increases the distances in the triple or brings it to the previous configuration. This implies that the initial quiver $Q$ was minimal.

Similarly to realizations by reflections (see Definition 2.2) we define realizations by $\pi$-rotations.

Definition 3.4 Let $B$ be a $3 \times 3$ skew-symmetric matrix. We say that a triple of vectors $\mathbf{v}=\left(v_{1}, v_{2}, v_{3}\right)$ is a geometric realization by $\pi$-rotations of $B$ if the assumptions of Lemma 3.1 hold. We also say that $\mathbf{v}$ provides a realization of the mutation class of $B$ if the mutations of $\mathbf{v}$ via partial $\pi$-rotations agree with all the mutations of $B$, i.e. if the assumptions of Lemma 3.1 hold after every sequence of mutations.

We can now formulate the following immediate corollary of Lemma 3.1.

Lemma 3.5 A mutation-acyclic quiver has no realization by $\pi$-rotations.

Theorem 3.6 Let $Q$ be a mutation-cyclic rank 3 quiver, and let $B$ be the corresponding skew-symmetric matrix. Then the mutation class of $B$ has a realization by reflections or a realization by $\pi$-rotations. 
Proof Since $Q$ is mutation-cyclic, Lemma 3.3 implies that $B=(p, q, r)$ with $p, q, r \geq 2$. If there is a triple of points on $\mathbb{H}^{2}$ on mutual distances $d_{p}, d_{q}, d_{r} \geq 0$, where $d_{x}=\operatorname{arccosh} \frac{x}{2}$, then Lemma 3.1 guarantees the realization by $\pi$-rotations (as $2 \cosh d(u, v)=-(u, v))$. Such a triple of points on $\mathbb{H}^{2}$ does exist if and only if the triangle inequality holds for $d_{p}, d_{q}, d_{r}$.

If we assume that the triangle inequality does not hold, then it is an easy exercise in hyperbolic geometry to find a triple of lines $l_{p}, l_{q}, l_{r}$ in $\mathbb{H}^{2}$ such that $d_{p}=d\left(l_{q}, l_{r}\right), d_{q}=d\left(l_{p}, l_{r}\right)$ and $d_{r}=d\left(l_{q}, l_{p}\right)$, and two of these are separated by the third one (as in Fig. 2, left). This provides us with a realization by reflections.

\section{Geometry Governed by the Markov Constant}

Definition 4.1 The Markov constant $C(p, q, r)$ for a triple $(p, q, r)$, where $p, q, r \in$ $\mathbb{R}$, was introduced by Beineke et al. (2011) as

$$
C(p, q, r)=p^{2}+q^{2}+r^{2}-p q r .
$$

For a cyclic quiver $Q$ with weights $p, q, r, C(Q)$ is defined as $C(p, q, r)$, while for an acyclic quiver with weights $p, q, r$ one has $C(Q):=C(p, q,-r)$ (this can be understood as turning an acyclic quiver into a cycle at the price of having a negative weight). It is observed in Beineke et al. (2011) that $C(Q)$ is a mutation invariant, it was also shown in Beineke et al. (2011) that in the case of integer weights $C(Q)$ characterizes (with some exceptions) the mutation-acyclic quivers:

Proposition 4.2 (Beineke et al. (2011), extract from Theorem 1.2) Let $Q$ be a rank 3 cyclic quiver with integer weights given by $p, q, r \in \mathbb{Z}_{\geq 0}$. Then the following conditions are equivalent.

(1) $Q$ is mutation-cyclic;

(2) $p, q, r \geq 2$ and $C(p, q, r) \leq 4$;

(3) $C(p, q, r)<0$ or $Q$ is mutation-equivalent to one of the following classes:

(a) $C(p, q, r)=0,(p, q, r)$ is mutation-equivalent to $(3,3,3)$;

(b) $C(p, q, r)=4,(p, q, r)$ is mutation-equivalent to $(q, q, 2)$ for some $q>2$.

Our next aim is to give a geometric interpretation of $C(Q)$ as well as to extend the result to the case of real numbers $p, q, r$.

Fig. 2 No realization by reflections for mutation-cyclic quivers (a)

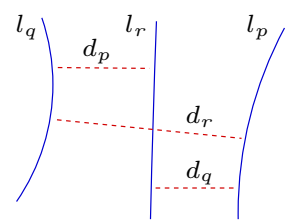

(b)

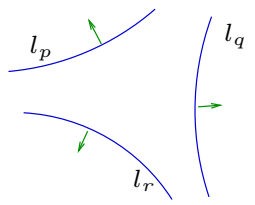


The question of recognizing whether a quiver $Q$ is mutation-acyclic is non-trivial if $Q$ is not acyclic itself [i.e. $Q$ is a cycle $(p, q, r)$ ] and if $p, q, r \geq 2$ [otherwise we just use Lemma 3.3(a)]. For quivers of this type, the proof of Theorem 3.6 shows that $Q$ can be realized by $\pi$-rotations (and is mutation-cyclic by Lemma 3.5) or by reflections depending on the triangle inequality for $d_{r} \leq d_{p}+d_{q}$, where $p \leq q \leq r$ and $d_{x}=\operatorname{arccosh} \frac{x}{2}$. Denote

$$
\Delta(Q)=d_{p}+d_{q}-d_{r}
$$

understanding $\Delta(Q) \geq 0$ as "triangle inequality holds" and $\Delta(Q)<0$ as "it does not".

Lemma 4.3 Let $Q=(p, q, r)$ be a rank 3 cyclic quiver with $2 \leq p \leq q \leq r$. Then

- if $\Delta(Q)>0$ then $C(Q)<4$;

- if $\Delta(Q)=0$ then $C(Q)=4$;

- if $\Delta(Q)<0$ then $C(Q)>4$.

Proof $\Delta(Q)<0$ if and only if $\cosh \left(d_{p}+d_{q}\right)<\cosh \left(d_{r}\right)$. Since $\cosh \left(d_{x}\right)=x / 2$, we have

$$
\begin{aligned}
\cosh \left(d_{p}+d_{q}\right) & =\frac{p}{2} \frac{q}{2}+\sinh \left(\operatorname{arccosh} \frac{p}{2}\right) \sinh \left(\operatorname{arccosh} \frac{q}{2}\right) \\
& =\frac{p q}{4}+\sqrt{\left(\frac{p^{2}}{4}-1\right)\left(\frac{q^{2}}{4}-1\right)} .
\end{aligned}
$$

Hence, $\Delta(Q)<0$ is equivalent to $\sqrt{\left(p^{2}-4\right)\left(q^{2}-4\right)}<2 r-p q$. Therefore, $\Delta(Q)<$ 0 implies $\left(p^{2}-4\right)\left(q^{2}-4\right)<(2 r-p q)^{2}$, i.e. $4<p^{2}+q^{2}+r^{2}-p q r=C(Q)$. An easy calculation shows that $C(Q)>4$ and $2 \leq p \leq q \leq r$ imply $2 r-p q>0$, so $C(Q)>4$ also implies $\Delta(Q)<0$.

Theorem 4.4 Let $Q$ be a rank 3 quiver with real weights. Then

(1) if $Q$ is mutation-acyclic then $C(Q) \geq 0$ and $Q$ admits a realization by reflections;

(2) if $Q$ is mutation-cyclic then $C(Q) \leq 4$ and $Q$ admits a realization by $\pi$-rotations;

(3) $Q$ admits both realizations (by reflections and by $\pi$-rotations) if and only if $Q$ is cyclic with $p, q, r \geq 2$ and $C(Q)=4$.

Proof (1) If $Q$ is mutation-acyclic, consider the acyclic representative (we may assume it is $Q$ itself). Then $C(Q) \geq 0$ as it is a sum of four non-negative terms. Existence of a realization by reflections is guaranteed by Corollary 2.3 .

(2) If $Q=(p, q, r)$ is mutation-cyclic, then by Lemma 3.3(a) we have $p, q, r \geq 2$, and by Theorem 3.6 $Q$ has a realization either by reflections in $\mathbb{H}^{2}$ or by $\pi$-rotations (again, in $\mathbb{H}^{2}$ ). Which of the options holds depends on the triangle inequality, i.e., on the sign of $\Delta(Q)$, which is determined by the sign of $4-C(Q)$. More precisely, if $C(Q) \leq 4$ then the triangle inequality holds and $Q$ has a realization by $\pi$-rotations, and if $\bar{C}(Q)>4$ then $Q$ has a realization by reflections. 
Suppose that a mutation-cyclic quiver $Q$ has $C(Q)>4$ and, hence, has a realization by reflections. It is shown in Section 5 of Beineke et al. (2011) that every mutationcyclic class with $C(Q) \neq 4$ contains a minimal element $Q_{\min }$, where the sum of the weights $p+q+r$ is minimal over the whole mutation class [notice that Beineke et al. (2011) shows this for all mutation classes with real weights]. Consider the realization of $Q_{\min }=\left(p_{\min }, q_{\min }, r_{\min }\right)$. As $Q_{\min }$ is still mutation-cyclic, we have $p_{\min }, q_{\min }, r_{\min } \geq 2$ which implies that the lines $l_{p}, l_{q}, l_{r}$ in the realization of $Q_{\min }$ do not intersect each other. If one of the lines (say, $l_{r}$ ) separates the others (see Fig. 2a), then partial reflection in $l_{r}$ (reflection of exactly one of $l_{p}$ and $l_{q}$ ) decreases one of the three distances, which contradicts the assumption that $Q_{\min }$ is minimal in the mutation class. If none of these lines separates the other two (see Fig. 2b), then for any choice of normal vectors there will be even number of positive scalar products $\left(v_{i}, v_{j}\right)$, which does not agree with Definition 2.2 for a cyclic quiver.

By Theorem 3.6, the contradiction shows that every mutation-cyclic quiver $Q$ has $C(Q) \leq 4$, admits a realization by $\pi$-rotations, and does not admit a realization by reflections if $C(Q) \neq 4$.

(3) First, by Lemma 3.5 a mutation-acyclic quiver cannot be realized by $\pi$-rotations. Next, a mutation-cyclic quiver with $C(Q) \neq 4$ cannot be realized by reflections as shown in the proof of part (2). Finally, suppose that $Q$ is mutation-cyclic and $C(Q)=4$. Then there is a realization of $Q$ by $\pi$-rotations about 3 collinear points (as $C(Q)=4$ is equivalent to the equality in the triangle inequality). Now, consider the line $l$ containing these three points. Taking three lines through these three points orthogonal to $l$ gives a realization by reflections.

Remark 4.5 (On realizations of $(2,2,2)$ ) In case of the quiver $(2,2,2)$ both realizations above are very degenerate (i.e., either reflections with respect to three coinciding lines or $\pi$-rotations with respect to three coinciding points). However, one can also consider a realization by reflections with respect to three mutually parallel lines (in $\mathbb{E}^{2}$ or $\mathbb{H}^{2}$ ), this will lead to an infinite group $G$.

Remark 4.6 As it is mentioned in Section 5 of Beineke et al. (2011), if $Q$ is mutationcyclic with $C(Q)=4$ then the mutation class of $Q$ may have no minimal quiver. Having in mind any of the two realizations of $Q$ described above, it is clear that a mutation-cyclic $Q=(p, q, r)$ has a minimal representative in the mutation class if and only if $d_{p} / d_{q} \in \mathbb{Q}$. If $d_{p} / d_{q} \notin \mathbb{Q}$ then we can always make the distances between three collinear points (or between three lines) as small as we want, which means that the quiver tends to the Markov quiver $(2,2,2)$.

Remark 4.7 (Geometric meaning of $C(Q)$ for mutation-acyclic $Q$ ) If $Q$ is mutationacyclic, $C(Q)$ is also responsible for the choice of the space $\mathbb{H}^{2}, \mathbb{E}^{2}$ and $\mathbb{S}^{2}$. Indeed, the choice of this space depends on the sign of the determinant of the matrix $M(B)$ (see Sect. 2.2.1), cf. Seven (2012): $\operatorname{det} M(B)=-2\left(p^{2}+q^{2}+r^{2}+p q r-4\right)=$ $-2(C(Q)-4)$.

Remark 4.8 (Geometric meaning of $C(Q)$ for mutation-cyclic $Q)$ Let $Q=(p, q, r)$ be mutation-cyclic, let $A, B, C \in \mathbb{H}^{2}$ be the points providing a realization of $Q$ by $\pi$-rotations, denote by $R_{A}, R_{B}, R_{C}$ the corresponding $\pi$-rotations. Then $C(Q)$ is 
Fig. 3 Geometric meaning of $C(Q)$

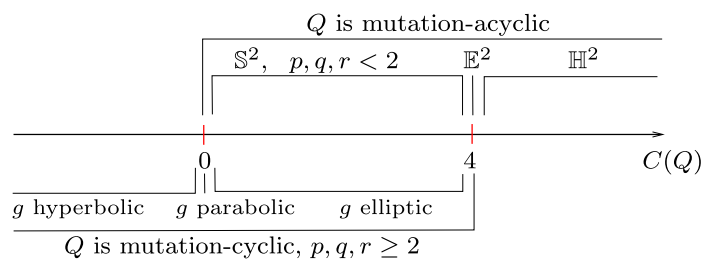

responsible for the type of the hyperbolic isometry $g=R_{A} \circ R_{B} \circ R_{C}$ in the following way: $g$ is elliptic (rotation by $\alpha$, where $2 \cos \alpha=\sqrt{4-C}$ ) if $C(Q)>0$, parabolic if $C(Q)=0$, and hyperbolic (translation by $d$, where $2 \cosh d=\sqrt{4-C}$ ) if $C(Q)<0$.

This can be proven following the ideas of Beardon $(1983, \S 11.5)$. To a triangle with sides $a, b, c$ and opposite angles $\alpha, \beta, \gamma$ one assigns a positive number $\lambda:=\sinh a \sinh b \sin \gamma=\sinh b \sinh c \sin \alpha=\sinh c \sinh a \sin \beta$, where the equality follows from the (hyperbolic) sine rule. Theorem 11.5.1 of Beardon (1983) states that the square of the trace of the element of $P S L(2, \mathbb{R})$ corresponding to $g=R_{A} \circ R_{B} \circ R_{C}$ equals $4 \lambda^{2}$. Then a short exercise in hyperbolic geometry shows that $2 \lambda=\sqrt{4-C}$, while the types of hyperbolic isometries are distinguished by the corresponding traces, see Beardon (1983).

We now summarize the geometric meaning of $C(Q)$ provided in Theorem 4.4 and Remarks 4.7, 4.8 in Fig. 3. Namely, $C(Q)$ tells whether $Q$ is mutation-acyclic or mutation-cyclic (admits realization by reflections or $\pi$-rotations), for realization by reflections it chooses the space where the group $G$ acts, and for realization by rotations tells the type of the product of the generators.

\section{Application: Classification of Rank 3 Quivers of Finite Mutation Type}

We now use the geometric models constructed above to classify rank 3 mutation-finite quivers.

Lemma 5.1 Let $Q=(p, q, r)$ or $Q=(p, q,-r)$ be a mutation-finite quiver, $p, q, r \in$ $\mathbb{R}_{\geq 0}$. Then $p, q, r \leq 2$.

Proof Suppose first that $Q$ is cyclic, i.e. $Q=(p, q, r)$, we may assume $p \geq q \geq r>$ 0 . If $p>2$, then $r^{\prime}=p q-r>2 q-r \geq q$, which implies that the mutation class contains an infinite sequence of quivers with strictly increasing sum of weights, so $Q$ cannot be mutation-finite.

Now, suppose $Q=(p, q,-r)$ is acyclic with $\max (p, q, r)>2$. Applying, if needed, sink/source mutations, we may assume $Q=(r, q,-p)$ with $p \geq q \geq r>0$, $p>2$. Then, after one more mutation we get a cyclic quiver with $p^{\prime}=q r+p>2$, which results in an infinite mutation class as shown above.

A combination of Lemma 3.3 with Lemma 5.1 leads to the following. 
Corollary 5.2 If $Q$ is a mutation-cyclic quiver of finite mutation type then $Q=$ $(2,2,2)$.

Thus, we only need to consider mutation-acyclic quivers, i.e., ones represented by reflections in one of the spaces $\mathbb{S}^{2}, \mathbb{E}^{2}$ and $\mathbb{H}^{2}$ (depending on the sign of $4-C(Q)$ ), see Theorem 4.4(1).

Lemma 5.3 Suppose that $Q=(p, q, \pm r)$ is mutation-finite. Then $p=2 \cos (\pi k / l)$ for some $k \in \mathbb{Z}_{\geq 0}, l \in \mathbb{Z}_{+}$. The same holds for $q$ and $r$.

Proof By Lemma 5.1 we have $p, q, r \leq 2$, so the lines $l_{p}, l_{q}$ and $l_{r}$ in the realization of $Q$ intersect each other forming some angles $\theta_{p}, \theta_{q}, \theta_{r}$ (if $p=2$ then the lines $l_{q}$ and $l_{r}$ are parallel).

Suppose $Q=(p, q, \pm r)$ and $p=2 \cos \theta_{p}$. Applying $\mu_{2}$ and $\mu_{1}$ alternately, we will get infinitely many triples of lines $\left(l_{p}^{(n)}, l_{q}^{(n)}, l_{r}^{(n)}\right)$ where $l_{p}=l_{p}^{(n)}$ and all lines $l_{q}^{(n)}, l_{r}^{(n)}$ pass through the same point $O=l_{q} \cap l_{r}$ and form the same angle $\theta_{p}=\angle\left(l_{q}^{(n)}, l_{r}^{(n)}\right)=\angle\left(l_{r}^{(n)}, l_{q}^{(n+1)}\right)$, see Fig. 4. If $\theta_{p}$ is not a rational multiple of $\pi$, then there are infinitely many intersection points of lines $l_{r}^{(n)}$ with $l_{p}$, thus infinitely many distinct angles. Therefore, quivers obtained from $Q$ by mutations $\mu_{2}$ and $\mu_{1}$ will contain infinitely many different entries, which implies that $Q$ cannot be mutationfinite.

Lemma 5.4 Let $Q$ be a mutation-acyclic quiver having a realization by reflections in $\mathbb{H}^{2}$ (i.e. $C(Q)>4$ ). Then $Q$ is not mutation-finite.

Proof By Lemma 5.1, we may assume $p, q, r \leq 2$, i.e. every quiver in the mutation class is represented by a triple $l_{p}, l_{q}, l_{r}$ of mutually intersecting (or parallel) lines. First, suppose $p=2$ (i.e. $\theta_{p}=0$ and $l_{q}$ is parallel to $l_{r}$ ). By assumption $C(Q)>4$, which implies that $l_{p}, l_{q}, l_{r}$ are not mutually parallel. Hence, after several mutations preserving $l_{p}$ we will get a triple of lines $\left(l_{p}, l_{q}^{(n)}, l_{r}^{(n)}\right)$ where $l_{p}$ is disjoint from $l_{q}^{(n)}$ and $l_{r}^{(n)}$, see Fig 5a. This contradicts Lemma 5.1.

Thus, $Q$ (and every quiver in its mutation class) is realized by a triple of mutually intersecting lines. The angles $\theta_{p}, \theta_{q}, \theta_{r}$ in the triangle representing $Q=(p, q, \pm r)$ are functions of $p, q, r: p=2 \cos \theta_{p}$ (same for $q$ and $r$ ). So, if $Q$ is mutation-finite then there is a smallest non-zero angle $\theta_{\min }$ such that $\theta_{\min }$ appears as an angle for a realization of some $Q^{\prime}$ in the mutation class of $Q$.
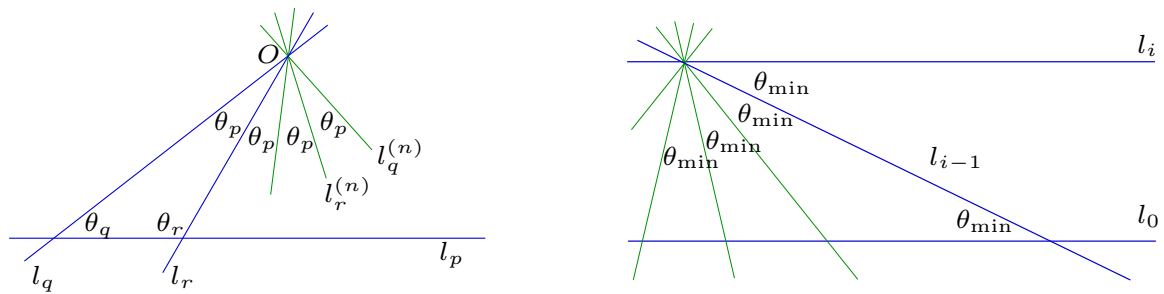

Fig. 4 Angles are $\pi$-rational in mutation-finite case 
(a)

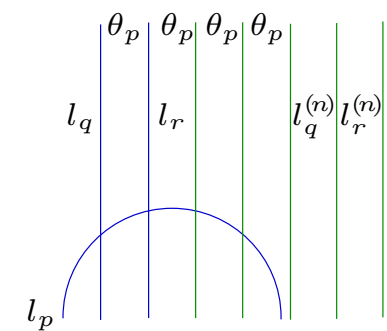

(b)

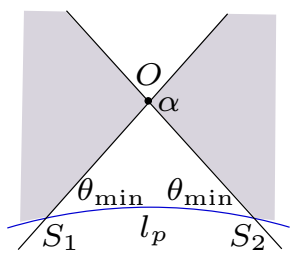

Fig. 5 Hyperbolic case. (We use upper half-plane model on the left and Poincaré disc model on the right)

Consider the realization $T_{0}=\left(l_{p}, l_{q}, l_{r}\right)$ of the quiver $Q^{\prime}$ and let $\theta_{\min }=\angle\left(l_{q}, l_{r}\right)$. Applying mutations $\mu_{2}$ and $\mu_{1}$ as in the proof of Lemma 5.3 (i.e., $l_{p}$ is always preserved and the image of $l_{q}$ is reflected with respect to the image of $l_{r}$ or vice versa), we will get further triangles $T_{i}$ realizing different quivers in the mutation class. We aim to show that some of the triangles $T_{i}$ either contains an angle smaller than $\theta_{\min }$ or has two disjoint sides (contradicting Lemma 5.1).

Let $O=l_{q} \cap l_{r}$. Consider the lines through $O$ forming the angle $\theta_{\min }$ with $l_{p}$ (see Fig. 5b), let $S_{1}$ and $S_{2}$ be the intersection points of these lines with $l_{p}$. Let $\alpha$ be the other angle formed by these lines (see Fig. 5b). Each of the triangles $T_{i}$ has $O$ as a vertex, and as the sum of angles in a hyperbolic triangle is less than $\pi$, we have $\angle S_{1} O S_{2}<\pi-2 \theta_{\min }$, which implies that

$$
\alpha=\pi-\angle S_{1} O S_{2}>2 \theta_{\min } .
$$

This means that at least one of the triangles $T_{i}$ will have a side crossing the grey domain between the lines. However, such a line will either be disjoint from $l_{p}$ or parallel to $l_{p}$ (contradicting Lemma 5.1 or the case considered above respectively), or it will cross $l_{p}$ at an angle smaller than $\theta_{\min }$ which is not possible either. The contradiction completes the proof of the lemma.

Lemma 5.5 Suppose that $Q$ is mutation-acyclic and has a realization by reflections in $\mathbb{E}^{2}$ (i.e. $C(Q)=4$ ). Then the following conditions are equivalent:

(a) $Q$ is mutation-finite;

(b) $Q=\left(p_{1}, p_{2}, \pm p_{3}\right)$ with $p_{i}=2 \cos \left(\pi t_{i}\right)$, where $t_{i} \in \mathbb{Q}$;

(c) $Q$ is mutation-equivalent to $(2 \cos (\pi / n), 2 \cos (\pi / n), 2)$, where $n \in \mathbb{Z}_{+}$.

Proof Condition (a) implies (b) by Lemma 5.3. Next, (b) says that in the realization $\left(l_{0}, l_{1}, l_{2}\right)$, one has $\angle\left(l_{1}, l_{0}\right)=k_{1} \pi / n_{1}$ and $\angle\left(l_{2}, l_{0}\right)=k_{2} \pi / n_{2}$ for some $k_{i}, n_{i} \in \mathbb{Z}_{+}$. This implies that under the mutations one can only obtain angles of size $k \pi / n_{1} n_{2}$, where $k \in \mathbb{Z}_{+}, k<n_{1} n_{2}$. So, in any quiver mutation-equivalent to $Q$ the weights can only take finitely many values $2 \cos \left(k \pi / n_{1} n_{2}\right)$, which results in finitely many quivers in the mutation class. This shows equivalence of (a) and (b). Obviously, (c) implies (b). We are left to show that (c) follows from either (a) or (b).

Assume $Q$ is mutation-finite. Then there is a minimal angle $\theta_{\min }$ obtained as an angle between the lines in a realization of some quiver $Q^{\prime}$ in the mutation class of 
$Q$. Assume that $\theta_{\min }=\angle\left(l_{1}, l_{2}\right)$ and consider the alternating sequence of mutations $\mu_{1}$ and $\mu_{2}$. Up to conjugation, we can assume that all these mutations preserve $l_{0}$ and reflect the image of $l_{2}$ with respect to the image of $l_{1}$ (or vice versa). We obtain finitely many lines $l_{1}, l_{2}, \ldots, l_{m}$ through $O=l_{1} \cap l_{2}$, any two adjacent lines $l_{i}$ and $l_{i+1}$ form an angle $\theta_{\min }$ and belong to a realization of one quiver (together with $l_{0}$ ). As the angle formed by $l_{0}$ and any of these lines cannot be smaller than $\theta_{\min }$, we conclude that $\theta_{\min }=\pi / n$ for some integer $n$, and one of $l_{1}, \ldots, l_{m}$, say $l_{i}$, is parallel to $l_{0}$, see Fig. 4 . Then the lines $\left(l_{i}, l_{i-1}, l_{0}\right)$ form a realization of some quiver $Q^{\prime \prime}$ in the mutation class of $Q$, where $Q^{\prime \prime}=\left(2 \cos \theta_{\min }, 2 \cos \theta_{\min }, 2\right), \theta_{\min }=\pi / n$. This shows that (a) implies (c).

Remark 5.6 An example of acyclic representative in the mutation class of $\left(2 \cos \frac{\pi}{n}\right.$, $\left.2 \cos \frac{\pi}{n}, 2\right)$ is $\left(2 \cos \frac{\pi}{n}, 2 \cos \left(\frac{\pi}{2}-\frac{\pi}{2 n}\right),-2 \cos \left(\frac{\pi}{2}-\frac{\pi}{2 n}\right)\right)$ if $n$ is odd and $\left(2 \cos \frac{\pi}{n}\right.$, $\left.2 \cos \left(\frac{\pi}{2}-\frac{\pi}{n}\right), 0\right)$ if $n$ is even.

Lemma 5.7 Suppose that $Q$ is mutation-acyclic and has a realization by reflections in $\mathbb{S}^{2}$ (i.e. $\left.C(Q)<4\right)$. If $Q$ is mutation-finite then $Q$ is mutation-equivalent to $\left(2 \cos \left(\pi t_{1}\right), 2 \cos \left(\pi t_{2}\right), 0\right)$, where $\left(t_{1}, t_{2}\right)$ is one of the following pairs:

$$
(1 / 3,1 / 3), \quad(1 / 3,1 / 4), \quad(1 / 3,1 / 5), \quad(1 / 3,2 / 5), \quad(1 / 5,2 / 5)
$$

Proof By Lemma 5.3, the weights of $Q$ are of the form $2 \cos \theta$, where $\theta$ is a rational multiple of $\pi$. We will apply a mutation $\mu$ to $Q$ and check whether $\mu(Q)$ still satisfies this condition.

More precisely, we can assume that $Q$ is acyclic and

$$
Q=(p, q,-r)=\left(2 \cos \frac{\pi t}{n}, 2 \cos \frac{\pi s}{n},-2 \cos \frac{\pi m}{n}\right),
$$

where $0<\frac{\pi t}{n} \leq \frac{\pi s}{n} \leq \frac{\pi m}{n} \leq \frac{\pi}{2}$ and $n \in \mathbb{Z}_{+}$such that $\pi / n$ is the smallest angle in the realization of the mutation class. Applying the mutation preserving $p$ and $q$ and changing $r$ to $r^{\prime}$ we get

$$
\begin{aligned}
r^{\prime} & =p q+r=4 \cos \frac{\pi t}{n} \cos \frac{\pi s}{n}+2 \cos \frac{\pi m}{n} \\
& =2 \cos \frac{\pi(s+t)}{n}+2 \cos \frac{\pi(s-t)}{n}+2 \cos \frac{\pi m}{n} .
\end{aligned}
$$

Notice that $r^{\prime}$ should be also a double cosine of an integer multiple of $\pi / n$. So, if $Q$ is mutation-finite, then there are integer numbers $s, t, m, k, n$ satisfying the equation

$$
\cos \frac{\pi(s+t)}{n}+\cos \frac{\pi(s-t)}{n}+\cos \frac{\pi m}{n}=\cos \frac{\pi k}{n} .
$$

It was shown by Conway and Jones (1976) that the only rational linear combinations of cosines of at most four rational multiples of $\pi$ between 0 and $\pi$ giving a rational 
number (without proper subset having this property) are the following:

$$
\begin{aligned}
& \cos \pi / 3=1 / 2, \quad \cos \pi / 2=0, \\
& -\cos \varphi+\cos (\pi / 3-\varphi)+\cos (\pi / 3+\varphi)=0(0<\varphi<\pi / 6), \\
& \cos \pi / 5-\cos 2 \pi / 5=1 / 2, \\
& \cos \pi / 7-\cos 2 \pi / 7+\cos 3 \pi / 7=1 / 2, \\
& \cos \pi / 5-\cos \pi / 15+\cos 4 \pi / 15=1 / 2, \\
& -\cos 2 \pi / 5+\cos 2 \pi / 15-\cos 7 \pi / 15=1 / 2,
\end{aligned}
$$

or one of four other equations, each involving four cosines on the left and $1 / 2$ on the right.

The latter four equations are irrelevant to us as they have too many terms to result in an equation of type (1). So, we need to consider the former seven equations and a trivial identity $\cos \varphi+\cos \psi=\cos \varphi+\cos \psi$. For each of these identities we match its terms to the terms of (1) (taking into account the signs of the terms) and compute the values of $s, t, m, k, n$. Most of the values obtained by this procedure are not relevant by one of the two reasons:

- either the values $s, t, m, n$ correspond to a triangle in $\mathbb{H}^{2}$ or $\mathbb{E}^{2}$, but not in $\mathbb{S}^{2}$ as needed;

- or the values $s, t, m, n$ do not correspond to an acute-angled triangle (which should be the case as we start with an acyclic quiver $Q$ ).

After removing irrelevant results, there are 13 cases left, some of them corresponding to mutation-infinite quivers. To exclude these, we check one more mutation and write an equation similar to (1) for $r q+p$ or $r p+q$. Removing these, we result in five quivers listed in the lemma plus two more quivers: $(1,1,-2 \cos 2 \pi / 5)$ and ( $2 \cos 2 \pi / 5,2 \cos 2 \pi / 5,-2 \cos 2 \pi / 5)$, which turned out to be mutation-equivalent to $(2 \cos \pi / 5,2 \cos 2 \pi / 5,0)$ and $(1,2 \cos 2 \pi / 5,0)$ respectively.

Remark 5.8 (Finite mutation classes, spherical case) In Table 1 we list the quivers belonging to the five finite mutation classes described by Lemma 5.7. Notice that two of these classes contain two acyclic representatives which are not sink/source equivalent.

Corollary 5.1 together with Lemmas 5.4, 5.5 and 5.7 imply the following classification.

Theorem 5.9 Let $Q$ be a connected rank 3 quiver with real weights. Then $Q$ is of finite mutation type if and only if it is mutation-equivalent to one of the following quivers:

(1) $(2,2,2)$;

(2) $(2 \cos (\pi / n), 2 \cos (\pi / n), 2), n \in \mathbb{Z}_{+}$;

(3) $(1,1,0), \quad(1, \sqrt{2}, 0), \quad(1,2 \cos \pi / 5,0), \quad(2 \cos \pi / 5,2 \cos 2 \pi / 5,0)$, $(1,2 \cos 2 \pi / 5,0)$. 
Table 1 Finite mutation classes with $C(Q)<4$

\begin{tabular}{lll}
\hline Acyclic quivers (up to sink/source) & Cyclic quivers & $C(Q)$ \\
\hline$(1,1,0)$ & $(1,1,1)$ & 2 \\
$(1, \sqrt{2}, 0)$ & $(\sqrt{2}, \sqrt{2}, 1)$ & 3 \\
$(1,2 \cos \pi / 5,0)$ & $(2 \cos \pi / 5,2 \cos \pi / 5,1)$ & $\frac{5+\sqrt{5}}{2}$ \\
$(2 \cos \pi / 5,2 \cos 2 \pi / 5,0)$ & $(2 \cos \pi / 5,2 \cos \pi / 5,2 \cos \pi / 5)$ & 3 \\
$(1,1,-2 \cos 2 \pi / 5)$ & $(2 \cos \pi / 5,2 \cos 2 \pi / 5,1)$ & \\
$(1,2 \cos 2 \pi / 5,0)$ & $(1,1,2 \cos \pi / 5)$ & $\frac{5-\sqrt{5}}{2}$ \\
$(2 \cos 2 \pi / 5,2 \cos 2 \pi / 5,-2 \cos 2 \pi / 5)$ & $(2 \cos 2 \pi / 5,2 \cos 2 \pi / 5,1)$ & \\
\hline
\end{tabular}
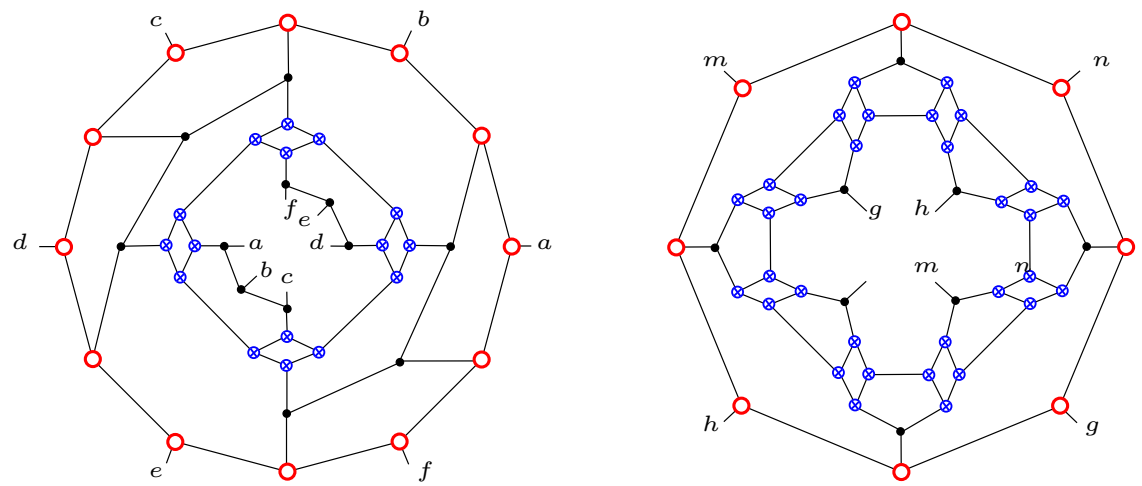

Fig. 6 "Exchange graphs" of the mutation classes for quivers $(2 \cos \pi / 5,2 \cos 2 \pi / 5,0)$ on the left and $(1,2 \cos 2 \pi / 5,0)$ on the right, the edges carrying the same letters should be identified. Each graph contains two connected acyclic belts labeled differently (blue and red) and can be drawn on a torus (color figure online)

Remark 5.10 The five mutation classes in part (3) of Theorem 5.9 contain all rank 3 quivers of "finite type", i.e. ones that can be modeled by reflections of finitely many vectors. Namely, the first three correspond to types $A_{3}, B_{3}$ and $H_{3}$, the exchange graphs for these classes can be found in Fomin and Reading (2007). The remaining two can also be modeled by reflections in some of the roots of the non-crystallographic root system $H_{3}$, we draw the corresponding "exchange graphs" in Fig. 6.

Remark 5.11 One can check that the triangular domains corresponding to quivers in the mutation classes of $A_{3}, B_{3}$ and $H_{3}$ tessellate the 2-sphere. The domains corresponding to quivers in the mutation classes of $(2 \cos \pi / 5,2 \cos 2 \pi / 5,0)$ and $(1,2 \cos 2 \pi / 5,0)$ tessellate a torus which is a two or fourfold covering of the sphere respectively.

\section{Acyclic Representatives in Infinite Real Mutation Classes}

Table 1 shows that there may be acyclic representatives in the same mutation class which differ much more than just by a sequence of sink/source mutations. 
Lemma 6.1 Let $Q=(p, q, r)$ be mutation-acyclic with $p<2$. Then, iterating mutations $\mu_{1}$ and $\mu_{2}$ (so that $p$ and $l_{p}$ are preserved), one can always reach an acyclic representative in at most $\left\lfloor\pi / \arccos \frac{p}{2}\right\rfloor$ mutations. In particular, there is an acyclic representative with weight $p$.

Proof Consider a realization $\left(l_{p}, l_{q}, l_{r}\right)$ of $Q$ by reflections and consider the triples of lines obtained from $\left(l_{p}, l_{q}, l_{r}\right)$ by mutations $\mu_{1}$ and $\mu_{2}$ applied alternately (see Fig. 4 ). If $n$ consecutive sectors cover the whole angle $2 \pi$ around the common point $O$ of $l_{q}$ and $l_{r}$, then at least one of the corresponding $\lfloor(n+1) / 2\rfloor$ triples is acute-angled.

Since $\arccos \frac{p}{2}=\theta_{p} \geq \frac{2 \pi}{n}$, we can take $n$ to be equal to $\left\lfloor 2 \pi / \arccos \frac{p}{2}\right\rfloor+1$. As one needs to make $\lfloor(n-1) / 2\rfloor$ mutations to obtain all the $\lfloor(n+1) / 2\rfloor$ triples that produce $n$ sectors covering $2 \pi$, the number of required mutations does not exceed $\left\lfloor\pi / \arccos \frac{p}{2}\right\rfloor$.

Theorem 6.2 Let $Q=(p, q, r)$ be mutation-acyclic with $0<C(Q)<4$. Then there exists an acyclic quiver $Q^{\prime}$ which can be obtained from $Q$ in at most $\left\lfloor\pi / \arcsin \frac{\sqrt{4-C(Q)}}{2}\right\rfloor$ mutations.

Proof Consider the realization $\left(l_{p}, l_{q}, l_{r}\right)$ of $Q$ by reflections. As $C(Q)<4$, this realization is a configuration of 3 lines on a sphere. By Lemma 6.1, it is sufficient to show that the angles in the realization of other quivers in the mutation class cannot be too small. We will show that they cannot become smaller than $\arcsin (\sqrt{4-C(Q)} / 2)$.

To show this we follow the same ideas as in the proof of Lemma 4.8. Namely, we choose a triangle bounded by $\left(l_{p}, l_{q}, l_{r}\right)$ and denote the lengths of its sides by $a, b, c$ and the opposite angles by $\alpha, \beta, \gamma$. Then we show that

$$
\lambda:=\sin a \sin \beta \sin \gamma=\sin b \sin \alpha \sin \gamma=\sin c \sin \beta \sin \gamma=\sqrt{4-C(Q)} / 2 .
$$

Here all but the last equalities follow from the spherical sine law, and the last equality follows from spherical second cosine law $\cos a \sin \beta \sin \gamma=\cos \beta \cos \gamma-\cos \alpha$, while taking in mind that $p=2 \cos \alpha, q=2 \cos \beta$ and $r=2 \cos \gamma$. In particular, we see that

$$
\sin \gamma \geq \sin a \sin \beta \sin \gamma=\sqrt{4-C(Q)} / 2 .
$$

As $C(Q)$ is independent on the representative in the mutation class, we have the same estimate for every angle in every triangle we can obtain by mutations of $\left(l_{p}, l_{q}, l_{r}\right)$.

Remark 6.3 There is no counterpart of Theorem 6.2 for the case of $C(Q) \geq 4$ (i.e. for Euclidean and hyperbolic realizations). Indeed, take any triple of lines $\left(l_{p}, l_{q}, l_{r}\right)$ in $\mathbb{E}^{2}$, where $l_{q}$ and $l_{r}$ form a $\pi$-irrational angle $\theta_{p}$. Then one can use mutations $\mu_{1}$ and $\mu_{2}$ to obtain a triple of lines with (at least one) arbitrary small angle. Repeating the same but now centered in the smallest angle, we can get a triple of lines with two angles arbitrary small (and thus the third one arbitrary close to $\pi$ ), i.e. a triple of almost coinciding lines. It is easy to see that this cannot be turned into an acute-angled configuration in a predefined number of mutations. 
Acknowledgements We would like to thank Philipp Lampe and Lutz Hille for stimulating discussions inspiring the current project. We are grateful to John Parker for referring us to the results of Conway and Jones (1976) and for a concise introduction to vanishing sums of roots of unity. We also thank Arkady Berenstein for sharing with us the results of Berenstein et al. (2006).

Open Access This article is distributed under the terms of the Creative Commons Attribution 4.0 International License (http://creativecommons.org/licenses/by/4.0/), which permits unrestricted use, distribution, and reproduction in any medium, provided you give appropriate credit to the original author(s) and the source, provide a link to the Creative Commons license, and indicate if changes were made.

\section{References}

Assem, I., Blais, M., Brüstle, T., Samson, A.: Mutation classes of skew-symmetric $3 \times 3$-matrices. Commun. Algebra 36(4), 1209-1220 (2008)

Barot, M., Geiss, C., Zelevinsky, A.: Cluster algebras of finite type and positive symmetrizable matrices. J. Lond. Math. Soc. (2) 73(3), 545-564 (2006)

Beardon, A.F.: The Geometry of Discrete Groups, vol. 91 of Graduate Texts in Mathematics. Springer, New York (1983)

Beineke, A., Brüstle, T., Hille, L.: Cluster-cyclic quivers with three vertices and the Markov equation. Algebra Represent. Theory 14(1), 97-112 (2011). With an appendix by Otto Kerner

Berenstein, A., Fomin, S., Zelevinsky, A.: Cluster algebras of rank 3. Unpublished drafts (2006)

Caldero, P., Keller, B.: From triangulated categories to cluster algebras. II. Ann. Sci. École Norm. Sup. (4) 39(6), 983-1009 (2006)

Conway, J.H., Jones, A.J.: Trigonometric Diophantine equations (On vanishing sums of roots of unity). Acta Arith. 30(3), 229-240 (1976)

Felikson, A., Tumarkin, P.: Mutation-finite quivers with real weights. arXiv e-prints. arXiv:1902.01997 (2019)

Felikson, A., Tumarkin, P.: Acyclic cluster algebras, reflection groups, and curves on a punctured disc. Adv. Math. 340, 855-882 (2018)

Felikson, A., Shapiro, M., Tumarkin, P.: Skew-symmetric cluster algebras of finite mutation type. J. Eur. Math. Soc. (JEMS) 14(4), 1135-1180 (2012)

Fock, V., Goncharov, A.: Moduli spaces of local systems and higher Teichmüller theory. Publ. Math. Inst. Hautes Études Sci. 103, 1-211 (2006)

Fomin, S., Reading, N.: Root systems and generalized associahedra. In: Geometric combinatorics, vol. 13 of IAS/Park City Math. Ser. Amer. Math. Soc., Providence, RI, pp. 63-131 (2007)

Fomin, S., Zelevinsky, A.: Cluster algebras. I. Foundations. J. Am. Math. Soc. 15(2), 497-529 (2002)

Fomin, S., Shapiro, M., Thurston, D.: Cluster algebras and triangulated surfaces. I. Cluster complexes. Acta Math. 201(1), 83-146 (2008)

Gekhtman, M., Shapiro, M., Vainshtein, A.: Cluster algebras and Weil-Petersson forms. Duke Math. J. 127(2), 291-311 (2005)

Lampe, P.: On the approximate periodicity of sequences attached to non-crystallographic root systems. Exp. Math. 27(3), 265-271 (2018)

Lee, K., Schiffler, R.: Positivity for cluster algebras. Ann. Math. (2) 182(1), 73-125 (2015)

Seven, A. I.: Mutation classes of $3 \times 3$ generalized Cartan matrices. In: Highlights in Lie algebraic methods, vol. 295 of Progr. Math., pp. 205-211. Birkhäuser/Springer, New York (2012)

Seven, A.I.: Cluster algebras and symmetric matrices. Proc. Am. Math. Soc. 143(2), 469-478 (2015)

Speyer, D., Thomas, H.: Acyclic cluster algebras revisited. In: Algebras, quivers and representations, vol. 8 of Abel Symp., pp. 275-298. Springer, Heidelberg (2013)

Warkentin, M.: Exchange graphs via quiver mutation. Ph.D. thesis, TU Chemnitz (2014)

Publisher's Note Springer Nature remains neutral with regard to jurisdictional claims in published maps and institutional affiliations. 